\title{
INTERCHANGEABILITY OF LOW-DOSE ORAL CONTRACEPTIVES
}

\author{
ARE CURRENT BIOEOUIVALENT TESTING MEASURES \\ ADEQUATE TO ENBURE THERAPEUTIC EQUIVALENCY?
}

\author{
Rudi Ansbacher, M.D., M.S. \\ Department of Obstetrics and Gynecology \\ University of Michigan Medical Center \\ Ann Arbor, Michigan 48109-0718 \\ Written while Scholar-in-Residence \\ Health Policy International \\ Princeton, New Jersey 08540
}

\begin{abstract}
Current Food and Drug Administration guidelines for assessing the differences in bioavailability between generic oral contraceptives and brand-name products are inadequate to ensure therapeutic equivalence. The guidelines do not take into account those women who may have blood levels of active ingredients well outside the range of acceptability. Due to the narrow therapeutic range of steroids, these women may become pregnant or experience an increased incidence of breakthrough bleeding. Furthermore, oral contraceptive packaging is unique to each manufacturer, and any change in brands (and therefore packaging) can easily negate the sequential administration of the appropriate tablet. These are among the reasons proposed for placing oral contraceptives in the critical drug category, in which generic substitution and interchangeability of products should not be allowed.
\end{abstract}

Submitted for publication September 5, 1990

Accepted for publication December 18, 1990 


\section{INTRODUCTION}

With the passage of the Drug Price Competition and Patent Term Restoration Act (Waxman-Hatch Amendment) 1 in 1984 and the acceptance of drug bioavailability measures as a prediction of therapeutic equivalence, generic copies of brand-name medications became widely used. Since that time, a number of questions have arisen concerning the therapeutic efficacy of the generics, the reliability of the products that are interchanged, and whether such product interchange is appropriate for the patient. These issues are discussed in this article using combination oral contraceptives as an example.

\section{FDA GUIDELINES}

The Food and Drug Administration (FDA) considers two products pharmaceutically equivalent if they contain the same active ingredients and are identical in strength, route of administration, and dosage form (one cannot substitute a capsule for a tablet). However, the two products may differ in their inert ingredients (binders, bulking agents, dyes, fillers, other excipients), color, flavor, shape, scoring configuration, packaging, shelf life, manufacturing variables (such as tablet compression force), and, within certain limits, labeling.

In the annual publication Approved Drug Products With Therapeutic Equivalence Evaluations, commonly referred to as the "Orange Book," "A" rated drug products are those "that the FDA considers to be therapeutically equivalent to other pharmaceutically equivalent products." "AB" rated drugs are those for which "actual or potential bioequivalence problems have been resolved with adequate in vivo and/or in vitro evidence supporting bioequivalence." oral contraceptives containing ethinyl estradiol in combination with norethindrone are rated "AB."

Ethingl estradiol is one of the 24 drugs that the FDA lists as having a narrow therapeutic range. 3 The rating for ethinyl estradiol in the "Orange Book" is "BP," a rating reserved for "drug products the FDA does not at this time consider to be therapeutically equivalent to other pharmaceutically equivalent products" with "active ingredients and dosage forms with potential bioequivalence problems." At the present time, "BP" rated drugs have not been tested for bioequivalency.

If, however, ethinyl estradiol is combined with a progestogen (such as norethindrone), the reting is "AB" because such combinations have been tested by current bioavailability measures. Are the standards used in these tests adequate to ensure therapeutic equivalence? 


\section{FORMULATIONS}

The currently marketed generic oral contraceptives, containing $35 \mathrm{mcg}$ ethinyl estradiol and $1 \mathrm{mg}$ norethindrone, are listed in (Table I).

TABLE I. Available oral contraceptives containing $35 \mathrm{mcg}$ ethinyl estradiol and $1 \mathrm{mg}$ norethindrone

\author{
Brand-Name \\ Norinyl $1+35$ \\ Ortho-Novum $1 / 35$ \\ Generic \\ Genora 1/35 \\ N.E.E. $1 / 35$
}

Nelova $1 / 35 E$

Norcept-E $1 / 35$

Norethin $1 / 35$
Manufacturer

Syntex Laboratories

ortho Pharmaceutical corp.

\section{Manufacturer}

Syntex Laboratories, distributed by Rugby (Dart) Laboratories

Schering Canada, Inc., distributed by Lexis Pharmaceuticals, Inc.

Watson, distributed by warnerChilcott (Parke-Davis)

Gedeon Richter, Ltd., distributed by Gyno Pharma, Inc.

Shiapparelli searle, distributed by G.D. Searle \& Co.

At one time Norinyl and ortho-Novum were identical. They were manufactured by the same company but distributed by two different companies. Therefore, they were completely interchangeable without the need for bioequivalence data. However, this may no longer be the case since their formulations now contain different inert ingredients 4 and no longer are manufactured in the same facility. Without bioequivalence data comparing these two current products, bioequivalence cannot be assumed, because changes made over the years may have caused differences in bioavailability of the active ingredients. 
CONTRACEPTION

\author{
BIOEQUIVALENCE
}

Under current FDA guidelines, 18 to 24 healthy women of reproductive age are used in a study designed to determine bioequivalence. Half of these women are given two tablets of the generic oral contraceptive and the other half receive two tablets of the brand-name product. (Twice the daily dose is necessary in order to assure assay sensitivity.) Blood levels of ethinyl estradiol and norethindrone are evaluated over a 24 to 48 hour period. One month later, in a cross-over design, each group is given the medication not taken previously, and blood levels of ethinyl estradiol and norethindrone are evaluated again.

Under present FDA guidelines, the $90 \%$ non-symmetric confidence interval limits for the mean parameter values, the area under the curve (AUC), and the maximum concentration ( $C_{\text {max }}$ ) of the generic product must fall between $80 \%$ and $120 \%$ of the values, for the brand-name product. These parameters are based on the plasma concentrations of the active hormonal ingredients of these medications.

These guidelines have a built-in margin for error, since a $\pm 5 \%$ variation in the amount of active ingredient between two individual pills manufactured by the same company is allowed. However, if the mean blood level of the active ingredient (ethinyl estradiol or norethindrone) in the group using the generic drug were $20 \%$ lower than in the group using the brand-name product, in some individuals, blood levels of these steroids would necessarily be below this $20 \%$ minimally acceptable value. These individuals could experience an unwanted pregnancy or an increased incidence of breakthrough bleeding. 5,6

\title{
CRITICAL DRUG CATEGORIES
}

In certain drug categories, minor changes in blood levels of the active ingredients may have a substantial impact on therapeutic outcome or clinical toxicity. The oral contraceptives, as presently formulated, function at their lowest effective dose to inhibit pregnancy and minimize hormonally related side effects. Estrogens have a narrow therapeutic range of action, and the estrogenic content of the low-dose oral contraceptives is in micrograms. This very low effective dose does not allow for typical margins of error in bioavailability, missed tablets, or improper consumption of the active/placebo sequences. Therefore, oral contraceptives should be considered for the critical therapeutic category for non-substitution.? 
The risks of increasing unwanted pregnancies do not justify the perceived cost of savings of generic drug substitution. cost containment is a worthy goal if safety and efficacy are not compromised. The choice should be based not on cost but on therapeutic effectiveness. Although generally not life threatening, an unwanted pregnancy can cause major emotional, physical, psychological, economic, and social problems for the individual woman, her family, and society in general.

In 1985, the cost of an uncomplicated vaginal delivery in the United States was $\$ 2923$, whereas a cesarean delivery without complications was $\$ 4862$ (both figures include the physician and hospital fees). raising a child to age 18 was then $\$ 53,000.9$ These estimates are even higher in 1990.

\section{OTHER CONSIDERATIONS}

\section{Balance}

The ratio of estrogen to progestogen, that is, hormonal balance, is critical, especially for the lower dose oral contraceptives, for the prevention of ovulation. 1 Furthermore, if the estrogen to progestogen ratio changes, increased side effects may result. Once titrated to optimal dosage with a particular dose and brand, a woman should continue using that product, since, at the present time, bioequivalence cannot be assumed under the advocated testing guidelines.

\section{Dosage}

The very low effective dose of these formulations has been finely tuned to minimize the hormonally related side effects. If the estrogen content of an oral contraceptive formulation falls below $30 \mathrm{mcg}$, and the progestogen content remains at $1 \mathrm{mg}$, breakthhrough bleeding and increased pregnancies may occur. 5,6 Moreover, individual variations in metabolism of the active hormones, especially the estrogenic component, can lead to lower blood levels of estrogen 11 , 12 ulting in non-effectiveness of the
product. 11 me must take into account the individual's response to the particular medication given. 


\section{CONTRACEPTION}

\section{Manufacturer to Manufacturer Consistency}

Variations in the excipients used by different manufacturers may affect the bioavailability of the active hormonal ingredients as well as the release profile of these hormones. Such differences may affect absorption, thereby changing the therapeutic effectiveness or causing an increase in side effects. 7

The FDA guidelines allow a $\pm 5 \%$ variation in the amounts of the active ingredients in individual pills, realizing that lot-to-lot variation occurs during production of the finished products. Such variations occur during the manufacturing process for both brand-name and generic products. Therefore, quality control is of utmost importance.

Not only is quality control a necessity during every step of the manufacturing process in order to eliminate error, but if a patient is switched to another formulation, close clinical observation is critical to ensure the safety and efficacy of the product.

\section{Packaging}

The packaging design, which facilitates the administration of the medication on a daily basis, is unique for each brand and generic product. Tablets containing the active hormonal ingredients have one color and placebo tablets have a different color, that is, they are color coded. One generic manufacturer's oral contraceptive placebo tablets are the same color as the active ingredient tablets of a brand-name product. In another generic package, similar in appearance to the brand-name package, the pili sequence was opposite to that in the brand-name package. Changing to another manufacturer's pill package can easily confuse the patient and negate the sequence control built into oral contraceptive packaging, even though special precautions are taken to instruct the patient.

Proper counseling by the pharmacist is a necessity. otherwise, the consumer can become confused, which could lead to lack of compliance, increased breakthrough bleeding, discontinuation of the pilis, and subsequent pregnancy.

\section{Pharmacy Product Selection}

The patient's confusion might be compounded by which package the pharmacy dispenses. With the various medications available (Table I), and the tendency of pharmacies to purchase their medications from different suppliers (depending on the currently lowest cost), it is not inconceivable that a woman might be using a different brand 
of oral contraceptive each month, especially if her prescription was written on a monthly basis and she were to use more than one pharmacy for her purchases.

\section{CONCLUSIONS}

Generic substitution should not be allowed for oral contraceptives, and the guidelines used to determine bioequivalency of this class of compounds (as compared with the majority of generic products) should be reevaluated because:

1. The formulations of generic oral contraceptives are different from that of the brand-name product, mainly in the excipients used.

2. Current testing guidelines for these narrow therapeutic range medications are not sufficient to assess the variability of blood levels in individual subjects and between subjects.

3. A subpotent tablet, or one which results in different blood levels than the original medication, can lead to unwanted pregnancy or breakthrough bleeding.

4. Differences in packaging designs of the various oral contraceptives have led to confusion when patients have been switched from a brand-name to a generic product. This can lead to lack of patient compliance, possibly resulting in an unwanted pregnancy.

\section{RECOMMENDATIONB}

Currently, a single-day test period is used to compare generic and brand-name oral contraceptives. Such results cannot be extrapolated for the entire 28-day cycle, since individual patient variation in the absorption of the active ingredients and the latter's subsequent metabolic breakdown cannot be assured; instead, reliance on the mean data from a number of subjects is used for comparison.

A more prolonged study period is necessary to ensure that the hormonal levels remain in the therapeutic range to prevent pregnancy throughout the critical ovulatory portion of the reproductive cycle. Even the manufacturers' patient product inserts have a disclaimer stating that the contraceptive reliance should not be placed on the product until after the first seven consecutive days of taking the medication. 


\section{CONTRACEPTION}

Alternate design studies of more than one day should be considered when testing formulations in which microgram doses of estrogen are combined with a milligram or less of progestogen. Replicate cross-over and multiple-dose studies are indicated. Steady-state study designs to ensure that the drug levels of estrogen and progesterone have stabilized would indicate the degree of absorption and metabolism of the hormones in an individual subject, and would determine differences between individuals. only then could the bioequivalence and interchangeability of these products be compared with accuracy.

To conduct even more meaningful bioequivalence and interchangeability studies, one should use a four-period, two-product, bio-replication cross-over design (where each product is given twice) in a steady-state to determine variations in responses from one product, within and between subjects, and between the two products.

Statistical testing should be uniform for all medications tested and should be based upon individual patient data, not mean data from a number of subjects. If the results do not fall into the ranges the FDA has published, public sale of the medication should not be allowed.

Post-marketing surveillance is necessary to ensure that all oral contraceptive products are therapeutically effective. If adverse reactions to a brand-name product occur, this is usually reported to the FDA, either directly or indirectly, by the manufacturer. However, when adverse drug reactions occur in association with switching from the brand-name to generic oral contraceptives, the threat of legal liability appears to have markedly decreased the reporting of such events.

When new products of identical active ingredient content are introduced into the marketplace, comparative testing of the innovator product and other similar approved products should be undertaken to assure equivalent therapeutic responses.

For these reasons, it is appropriate to place oral contraceptives in the critical drug category, in which generic substitution should not be allowed. 


\section{REFERENCES}

1. Drug Price competition and Patent Term Restoration Act. The Congressional Record. Washington, DC, September 12 , 1984, 5-10981.

2. Approved Drug Products with Therapeutic Equivalence Evaluations. 9 th ed. US Department of Health and Human Services, Public Health Service, Food and Drug Administration, Center for Drug Evaluation and Research, Office of Management, 1989.

3. FDC Reports, December 11, 1988:3.

4. Physicians' Desk Reference. 43rd ed. Oradell, NJ: Medical Economics Company, Inc., 1989:1505,2131.

5. Smith MA, Youngkin EQ. Current perspectives on combination oral contraceptives. Clin Pharm $1984 ; 3: 485-96$.

6. Koetsawang $S$, Kiriwat 0 , Sheth A, et al. A randomized, double-blind study of six combined oral contraceptives. WHO Task Force on oral Contraceptives $1982 ; 25(3): 231-$ 41.

7. Colaizzi JL, Lowenthal DT. Critical therapeutic categories: A contraindication to generic substitution? Clin Ther $1986 ; 8: 370-79$.

8. Gold RB, Kenney AM, Singh S. Paying for maternity care in the United States. Fam Plan Persp 1987;19:190-206.

9. Data from Center for Population Options, Washington, DC.

10. Frazer IS, Jansen RPS. Why do inadvertent pregnancies occur in oral contraceptive users? Contraception $1983 ; 27: 531$.

11. Helton ED, Williams MC, Goldzieher JW. Human urinary and liver conjugates of 17 alpha-ethynylestradiol. steroids 1976:27:851-67.

12. Helton ED, Simmons R, Meltz ML, Goldzieher JW. Variability in conjugates of ethynylestradiol produced by in vitro incubation of human liver tissue. Contraception $1977 ; 16: 257-60$. 